\title{
An Efficient Algorithm to Identify Minimal Failure-Causing Schemas from Exhaustive Test Suite
}

\author{
Yuanchao Qi ${ }^{1}$, Qi Wang ${ }^{1}$, Chiya $\mathrm{Xu}^{1}$, Tieke $\mathrm{He}^{2}$, and Ziyuan Wang ${ }^{1 *}$ \\ ${ }^{1}$ School of Computer Science and Technology, Nanjing University of Posts and Telecommunications, Nanjing, China \\ ${ }^{2}$ State Key Lab for Novel Software Technology, Nanjing University, Nanjing, China \\ *Corresponding: wangziyuan@ njupt.edu.cn
}

\begin{abstract}
Combinatorial testing is widely used to detect failures caused by interactions among parameters for its efficiency and effectiveness. Fault localization plays an important role in this testing technique. And minimal failure-causing schema is the root cause of failure. In this paper, an efficient algorithm, which identifies minimal failure-causing schemas from existing failed test cases and passed test cases, is proposed to replace the basic algorithm with worse time performance. Time complexity of basic and improved algorithms is calculated and compared. The result shows that the method that utilizes the differences between failed test cases and passed test cases is better than the method that only uses the sub-schemas of those test cases.
\end{abstract}

Keywords-Combinatorial testing, fault localization, minimal failure-causing schema, algorithm.

\section{INTRODUCTION}

Softwares may be affected by the interactions among its parameters. These interactions need to be tested to guarantee the quality of software. But for the software with $k$ parameters, it is unacceptable to cover all the possible $k$-tuple combinations of parametric values. Combinatorial testing provides a tradeoff between the testing cost and the degree of combinatorial coverage. It has been widely used for its efficiency and effectiveness, especially, in highly-configurable systems [1]. Test case generation and fault localization based on failurecausing schemas are hot areas of research.

Many researches focus primarily on generating test cases to filter suspicious failure-causing schemas. But there are few materials to discuss how to filter them. The default option is to construct all possible suspicious failure-causing schemas from failed test cases and filter them by passed test cases. In this paper, we propose an improved algorithm with better time performance. The analysis of time complexity shows the advantage of proposed algorithm. And the efficient algorithm has been applied in our practice to compute minimal failurecausing schemas for boolean-specification testing and Siemens program suite.

\section{BACKGROUND}

The model of minimal failure-causing schema was proposed by Nie et. al [2].

For a software system with $k$ parameters, we suppose each parameter $f_{i}$ has $a_{i}(1 \leq i \leq k)$ discrete valid values. Let $F=\left\{f_{1}, f_{2}, \ldots, f_{k}\right\}$ denote the set of parameter, and $V_{i}=$

DOI reference number: 10.18293/SEKE2016-233 $\left\{0,1, \ldots, a_{i-1}\right\}(i=1,2, \ldots k)$ the value set for $f_{i}$ without loss of generality.

Definition 1. (Schema). A $k$-tuple $s=\left(-, \ldots,-, v_{i_{1}},-, \ldots,-, v_{i_{2}}\right.$, $\left.-, \ldots,-, v_{i_{\tau}},-, \ldots,-\right)$ is a schema with strength $\tau$, or a $\tau$-way schema (or $\tau$-schema for short) $(1 \leq \tau \leq k)$. Where $\tau$ values are fixed as $v_{i_{1}} \in V_{i_{1}}, v_{i_{2}} \in V_{i_{2}}, \ldots, v_{i_{\tau}} \in V_{i_{\tau}}$, and other $k-\tau$ values are not fixed and represented as "-".

Definition 2. (Sub-schema and parent-schema). Schemas $s_{1}=$ $\left(v_{1}, v_{2}, \ldots, v_{k}\right)$ and $s_{2}=\left(v_{1}^{\prime}, v_{2}^{\prime}, \ldots, v_{k}^{\prime}\right)$ are $\tau_{1}$-schema and $\tau_{2}$ schema respectively $\left(\tau_{1} \leq \tau_{2}\right)$. If $\forall 1 \leq i \leq k,\left(v_{i}=-\right) \vee\left(v_{i}=\right.$ $\left.v_{i}^{\prime}\right)$ is true, then $s_{1}$ is a sub-schema of $s_{2}$, and $s_{2}$ is a parentschema of $s_{1}$. It is denoted as $s_{1} \prec s_{2}$. Especially, if $s_{1} \neq s_{2}$, then $s_{1}$ is a real sub-schema of $s_{2}$, and $s_{2}$ is a real parentschema of $s_{1}$.

Definition 3. (Failure-causing schema). A schema $s$ is a failure-causing schema (or $F S$ for short), if $\forall t \in T_{\text {all }}=$ $V_{1} \times V_{2} \times \ldots \times V_{k}, s \prec t \Rightarrow t$ is failed test case.

Definition 4. (Minimal failure-causing schema). A failurecausing schema $s$ is a minimal failure-causing schema (or $M F S$ for short), if any real sub-schema of $s$ is not a failurecausing schema.

\section{AlgORITHMS}

People pay more attention to the problem that how to identify minimal failure-causing schemas accurately. However, efficiency is also a fundamental issue. In this section, we will introduce the most used basic algorithm and then propose an improved one with better time complexity.

\section{A. Basic algorithm}

The basic algorithm, which identifies minimal failurecausing schemas from failed test cases and passed test cases, was mentioned in many materials. But its detailed process was often omitted. Here we describe the basic algorithm and analyze its time performance.

For each failed test case $t$, there are $C_{k}^{1} 1$-way sub-schemas, $C_{k}^{2}$ 2-way sub-schemas,..., and $C_{k}^{k} k$-way schemas.

When filtering suspicious schemas, from $n f \times C_{k}^{1} 1$-way sub-schemas of failed test cases will be filtered by $n p \times C_{k}^{1}$ 1-way sub-schemas of passed test cases, to $n f \times C_{k}^{k} k$-way sub-schemas of failed test cases be filtered by $n p \times C_{k}^{k} k$-way sub-schemas.

When comparing two $i$-way schemas $(i=1,2, \ldots, k)$, the values of $i$ parameters should be compared. Therefore, the total 


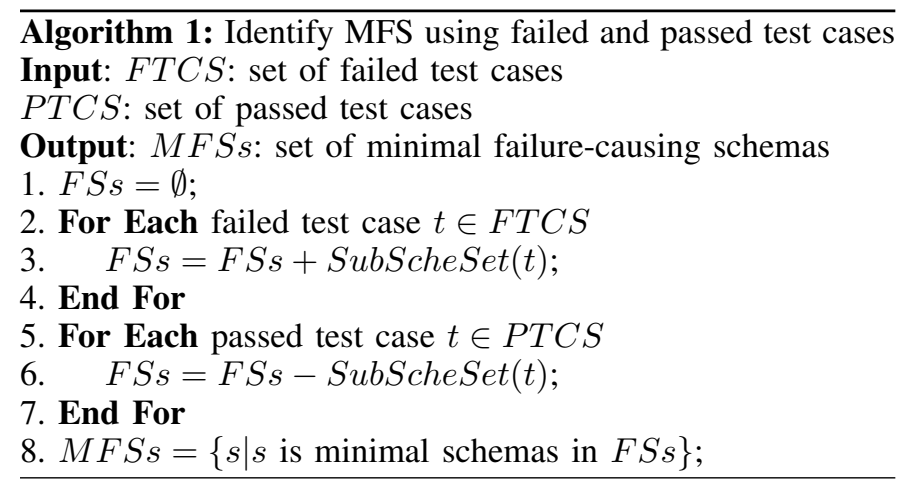

time complexity of filtering suspicious schemas in Algorithm 1 should be:

$$
\begin{aligned}
& O\left(n p \times C_{k}^{1} \times n f \times C_{k}^{1} \times 1+n p \times C_{k}^{2} \times n f \times C_{k}^{2} \times 2+\ldots\right. \\
&\left.+n p \times C_{k}^{k} \times n f \times C_{k}^{k} \times k\right) \sim O\left(n p \times n f \times \sum_{i=1}^{k}\left(i \times\left(C_{k}^{i}\right)^{2}\right)\right) .
\end{aligned}
$$

Additionally, in the process of selecting minimal ones from the set of failure-causing schemas, we can filter $\tau$-way failurecausing schemas by $(\tau-1)$-way's $(\tau=2,3, \ldots, k)$, for each failed test case. So there are totally $C_{k}^{k} \times C_{k}^{k-1}+C_{k}^{k-1} \times$ $C_{k}^{k-2}+\ldots+C_{k}^{k-2} \times C_{k}^{1}$ parametric values should be checked for each failed test case. Here note that $O\left(\sum_{i=2}^{k}\left(C_{k}^{i} \times C_{k} i-1\right)\right) \sim$ $O\left(\sum_{i=2}^{k}\left(C_{k}^{i}\right)^{2}\right) \sim O\left(\sum_{i=1}^{k}\left(C_{k}^{i}\right)^{2}\right) \sim O\left(C_{2 k}^{k}\right)$.

Therefore, the time complexity of the whole Algorithm 1 should be: $O\left(n p \times n f \times \sum_{i=1}^{k}\left(i \times\left(C_{k}^{i}\right)^{2}\right)+n f \times C_{2 k}^{k}\right)$.

\section{B. Improved algorithm}

Factually, the process of extracting and filtering suspicious schemas in the basic algorithm could be optimized to enhance its time performance. We will propose an improved algorithm by utilizing the differences between failed test cases and passed test cases.

Considering a failed test case $t$ and a passed test case $t^{\prime}$ we could construct a set of parameteres Diff_Param $\left(t, t^{\prime}\right)$ that contains all parameters whose parametric values in $t$ and $t^{\prime}$ are different. So, the process to identify MFS is described in Algorithm 2.

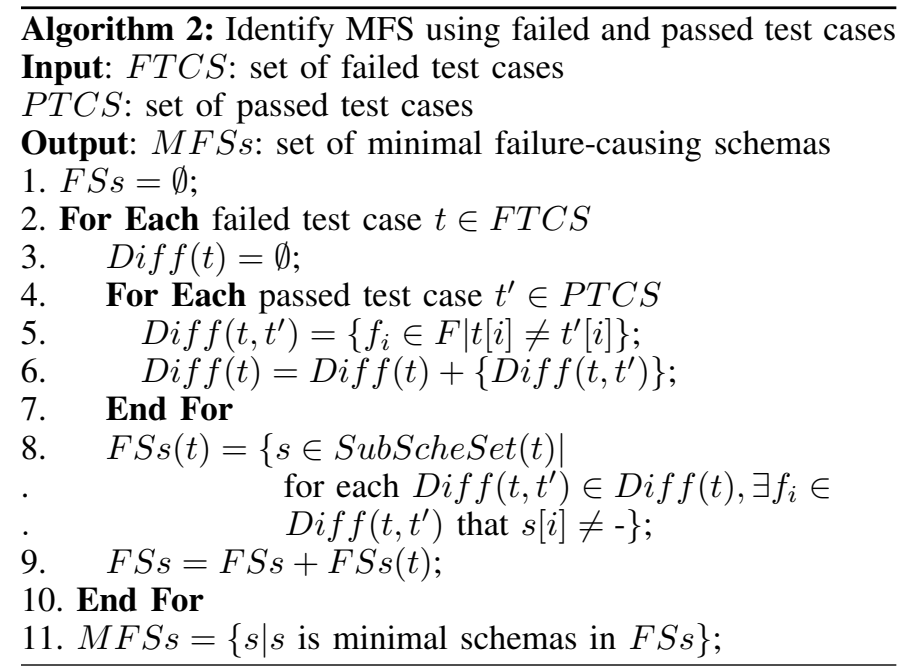

For a failed test case $t$, there are $k$ parametric values which should be checked when constructing a Diff_Param $\left(t, t^{\prime}\right)$ with the passed test case $t^{\prime}$. If there are $n p$ passed test cases, it is $n p \times k$. So there are totally $n p \times n f \times k$ parametric values which should be checked when constructing these sets for all failed test cases.

For a failed test case $t$, when selecting its failure-causing sub-schemas, there are $n p$ different Diff_Param $\left(t, t^{\prime}\right)$ to be checked. So there are totally $n p \times \sum_{i=1}^{k}\left(i \times C_{k}^{i}\right)$ parametric values which should be checked for one failed test case, and totally $n p \times n f \times \sum_{i=1}^{k}\left(i \times C_{k}^{i}\right)$ parametric values should be checked for all failed test cases.

Therefore, the total time complexity of selecting all failurecausing schemas in Algorithm 2 should be:

$$
\begin{aligned}
& O\left(n p \times n f \times k+n p \times n f \times \sum_{i=1}^{k}\left(i \times C_{k}^{i}\right)\right) \\
& \sim O\left(n p \times n f \times \sum_{i=1}^{k}\left(i \times C_{k}^{i}\right)\right)
\end{aligned}
$$

Since the time complexity of selecting minimal failurecausing schemas is $O\left(C_{2 k}^{k}\right)$ for each failed test case, the time complexity of the whole Algorithm 2 should be: $O(n p \times n f \times$ $\left.\sum_{i=1}^{k}\left(i \times C_{k}^{i}\right)+n f \times C_{2 k}^{k}\right)$.

\section{DISCUSSION}

\section{A. Outputs of Two Algorithms}

Algorithm 1 and Algorithm 2 obtain the same outputs for the same inputs. It is clear in the description of two algorithms, especially in the description of improved one.

\section{B. Comparing Time Performance}

According to the binomial theorem, $C_{2 k}^{k}=\sum_{i=1}^{k}\left(C_{k}^{i}\right)^{2}$. Since there are:

$$
\begin{aligned}
& \sum_{i=1}^{k}\left(C_{k}^{i}\right)^{2}<\sum_{i=1}^{k}\left(i \times\left(C_{k}^{i}\right)^{2}\right) \\
& \sum_{i=1}^{k}\left(i \times C_{k}^{i}\right)<\sum_{i=1}^{k}\left(i \times\left(C_{k}^{i}\right)^{2}\right)
\end{aligned}
$$

So it is obvious that $O\left(n p \times n f \times \sum_{i=1}^{k}\left(i \times C_{k}^{i}\right)+n f \times C_{2 k}^{k}\right)<$ $O\left(n p \times n f \times \sum_{i=1}^{k}\left(i \times\left(C_{k}^{i}\right)^{2}\right)+n f \times C_{2 k}^{k}\right)$. It is means that the time complexity of Algorithm 2 is less than that of Algorithm 1. Then we can make a conclusion that Algorithm 2 is better than Algorithm 1.

\section{CONCLUSION}

In this paper, we carefully study two algorithms that could identify MFSs by utilizing failed test cases and passed test cases. The time complexity of two algorithms shows that the approach which utilizes the difference between every failed test case and passed test case is clearly better than the other one. We believe that the research will improve the effectiveness and efficiency of practical testing.

\section{REFERENCES}

[1] C. Nie, H. Leung. A survey of combinatorial testing. ACM Computing Surveys (CSUR), 2011, 43(2): 11.

[2] C. Nie, H. Leung. The Minimal Failure-causing Schema of Combinatorial Testing. ACM Transactions on Software Engineering and Methodology (TOSEM), 2011, 20(4): 15. 